\title{
LRRK2 is expressed in areas affected by Parkinson's disease in the adult mouse brain
}

Javier Simón-Sánchez ${ }^{1}$, Vicente Herranz-Pérez ${ }^{1}$, Francisco OluchaBordonau $^{2}$, Jordi Pérez-Tur ${ }^{1, *}$

1. Unitat de Genètica Molecular. Departament de Genòmica i Proteòmica. Institut de Biomedicina de València-CSIC.

2. Departament d'Anatomia i Embriologia Humana. Facultat de Medicina. Universitat de València-Estudi General.

*: Address for correspondence at:

Unitat de Genètica Molecular.

Institut de Biomedicina de València-CSIC.

C/ Jaume Roig, 11. E46010 València (Spain).

Telephone: $\quad+34963391755$

Fax: $\quad+34963393774$

e-mail: $\quad$ jpereztur@ibv.csic.es

Running title: Expression of LRRK2 in the Adult Mouse Brain pages; 4 figures; 2 tables.

Word count: manuscript: 4,314 words; abstract: 179 words; introduction: 515 words. 


\section{ABSTRACT}

The LRRK2 gene was recently found to have multiple mutations that are causative for autosomal dominant inherited Parkinson's disease (PD). Previously, we used Northern blot analysis to show that that this gene was expressed in the cerebellum, cerebral cortex, medulla, spinal cord, occipital pole, frontal lobe, temporal lobe and caudate putamen. However, a more comprehensive map of LRRK2 mRNA localization in the central nervous system is still lacking. In this study we have mapped the distribution of the mRNA encoding for LRRK2 using non-radioactive in situ hybridization. We detected a moderate expression of this PD-related gene throughout the adult mouse brain. A stronger hybridization signal was observed in deep cerebral cortex layers, superficial cingulate cortex layers, the piriform cortex, hippocampal formation, caudate putamen, substantia nigra, the basolateral and basomedial anterior amygdala nuclei, reticular thalamic nucleus and also in the cerebellar granular cell layer. Given that LRRK2 mRNA is highly enriched in motor systems and also is expressed in other systems, we may conclude that mutations in LRRK2 may affect several motor and nonmotor structures that may play an important role in the development of PD. 


\section{INTRODUCTION}

Parkinson's disease (PD) is the second most common neurodegenerative disorder, affecting $2 \%$ of people over 65 years of age. Although the majority of PD cases are sporadic, a small percentage is caused solely by mutations in several recently identified genes. Despite their low frequency, genes that are causative for PD are extremely important since they can highlight the mechanisms and pathways leading to the onset of the disease, and foster the identification of new forms of therapeutic treatment.

The familial variant of PD can be divided into autosomal dominant and autosomal recessive forms. The currently identified autosomal dominant forms of PD can be due to mutations in the genes encoding $\alpha$-synuclein ( $S N C A$, PARK1), Ubiquitin CarboxylTerminal esterase L1 (UCH-L1, PARK5) or Dardarin (LRRK2, PARK8); while the autosomal recessive variants are caused by mutations in the genes encoding Parkin (PRKN, PARK2), DJ-1 (DJ-1, PARK7) and PTEN inducible kinase 1 (PINK-1, PARK6).

Recently our group identified four families from the Basque region in Spain with the autosomal dominant PD (Paisan-Ruiz et al., 2005). A multipoint linkage analysis showed us that these four families were linked to the PARK8 locus (12p11.2q13.1) described by Funayama et al. (2002). By means of systematic sequence analysis, we identified segregating mutations in all four Basque families within a $9 \mathrm{~kb}$ predicted transcript (Paisan-Ruiz et al., 2004). The approved symbol for the gene, LRRK2, is due to the presence of both the leucine-rich and MAPKKK domains in the protein. The protein is known as Dardarin, a word that originated from the Basque word dardara meaning "tremor". While the expression patterns of previously identified genes such as SNCA, PRKN, UCHL-1 and DJ-1 have been reported, those for LRRK2 are yet to be investigated.

An in silico analysis of dardarin using the SMART web site (http://smart.emblheidelberg.de) suggested that dardarin is a complex protein belonging to the ROCO kinases superfamily (Bosgraaf and Van Haastert, 2003). Proteins belonging to the ROCO superfamily stand out because of the existence of two conserved domains: a Roc domain (Ras on Complex Proteins) and a COR domain (C-terminal Of Roc). Ras-like domains are often involved in rearrangements of actin cytosqueleton, and they also play an important role in Ras-mediated cell transformation, cytokinesis, focal adhesion formation and MAPK pathway stimulation. In addition to these, a further three domains 
are also found in dardarin: Leucine Rich Repeats (LRR), a Tyrosine catalytic domain and a WD40 domain.

Our analysis of gene expression by Northern blot previously indicated that $L R R K 2$ gene is expressed in cerebellum, cerebral cortex, medulla, spinal cord, occipital pole, frontal lobe, temporal lobe and putamen (Paisan-Ruiz et al., 2004). However, a complete map of LRRK2 mRNA localization in the central nervous system is still lacking. The objective of this study is to provide a complete atlas of LRRK2 mRNA distribution in the adult mouse brain. 
MATERIALS AND METHODS

\section{In situ hybridization}

This work is based on five B2B6 adult male mice. All animal manipulations were approved by the Animal Care and Ethics Committee of the CSIC and the School of Medicine of the University of Valencia. Animals were ether-anesthetized and decapitated. Brains were quickly removed and immersed overnight in a fixative containing $4 \%$ paraformaldehyde in $0.1 \mathrm{M} \mathrm{pH} 7.4$ phosphate buffered saline- $0.01 \%$ diethyl pyrocarbonate (PBS-DEPC). After immersing the fixed brains in gelatineglutaraldehyde for 30-60 minutes, a coronal serial of 50 $\mathrm{mm}$ thick coronal sections were prepared using a vibratome (VT1000M, Leica).

Chromogenic in situ hybridization was performed as described elsewhere. Briefly, coronal sections of adult B2B6 mice were dehydrated and then rehydrated in a graded methanol series. Next, they were treated with proteinase $K$ and fixed in paraformaldehyde. After this treatment, the sections were prehybridized for $2-16 \mathrm{~h}$ at $50^{\circ} \mathrm{C}$ prior to incubating them for $3 \mathrm{~h}$ at $57^{\circ} \mathrm{C}$ with the digoxigenin-labelled probe. After removal of non-specific binding by sequential washes of decreasing concentrations of $\mathrm{SSC}$, the presence of the LRRK2 mRNA was revealed using an HRP-conjugated antidigoxigenin antibody and a chromogenic reaction using nitroblue tetrazolium chloride and 5-bromo-4-chloro-3-indolyl phosphate (all reagents from Roche Diagnostics). All reagents and materials were treated as necessary to ensure the absence of RNAse contamination.

\section{Probe synthesis}

Two in situ hybridization probes were synthesized by means of PCR amplification. The probes were amplified from a mouse cDNA library (Invitrogen) that recognized a fragment of the $L R R K 2$ gene from codon 1004 to codon 1172 (probe I) or a fragment from codon 2013 to codon 2171 (probe II). The oligonucleotides used for this amplification were the following:

LRRK2-PROBE1-F: 5'-ATCATCTAGAGTTGCCTCAGTAGCCACC-3'. LRRK2-PROBE1-R: 5'-ATCAGTCGACAAGGTAAGGCAGGCATTG-3'. LRRK2-PROBE2-F: 5'-TACGTCTAGAGCGAAGATTGCGGACTAC-3'. LRRK2-PROBE2-R: 5'-CACAGATATCCCAACCAGAGAGTCGCAC-3'. 
The PCR conditions used were two minutes at $95^{\circ} \mathrm{C}$ followed by thirty-five cycles of thirty seconds at $95^{\circ} \mathrm{C}$, thirty seconds at $58^{\circ} \mathrm{C}$ and one minute at $72^{\circ} \mathrm{C}$. After cycling, a ten-minute step at $72^{\circ} \mathrm{C}$ was performed. For this amplification $P f u$ turbo polymerase was used as per manufacturer's instructions (Stratagen). The amplified product was resolved on a $\%$ agarose-TBE gel, extracted the Minilute Gel extraction kit (Qiagen) and cloned into the $\mathrm{Xba}$ I-Sal I sites of the pBluescript KS-II' vector (Stratagene) using the Rapid ligation kit (Roche). The probes were synthesized by linearizing the construct with either Xba I (antisense probe) or Sal I (sense probe) followed by in vitro transcription with digoxigenin labelled nucleotides using an RNA oligonucleotide synthesis kit (Roche). To avoid secondary structure formation, RNA synthesized probes were diluted with $50 \%$ formamide. Both probes yielded equivalent results, only those for probe I are shown.

\section{Relative regional expression.}

To determine the level of the LRRK2 expression in different areas of the brain we performed several experiments with different development times (from 2 to $16 \mathrm{~h}$ ). The level of expression was then correlated with the time needed to obtain clear staining in each of the areas shown in the table. When a clear staining was observed in a particular area during the first $2 \mathrm{~h}$ of developing, it was assigned a "+++" mark, those areas that were clearly stained after $9 \mathrm{~h}$ of chromogenic reaction were assigned as "++" whereas those stained after $16 \mathrm{~h}$ of staining were designated with "+". Those which revealed no staining after $16 \mathrm{~h}$ were considered as not being able to express $L R R K 2$ or at least their level of expression was not detectable with this particular technique. 


\section{RESULTS}

We studied the LRRK2 mRNA expression in adult mice brains through in situ hybridization analysis using digoxigenin-labelled RNA probes. We first confirmed the specificity of the probe by comparing the staining pattern of the antisense probe with that of the sense probe. The antisense probe showed specific signal patterns, whereas no signal was observed with the sense probe. This difference between both probes indicates that the antisense probe can specifically hybridize with its corresponding transcript (Fig. $1)$.

As a result we found that $L R R K 2$ mRNA was expressed throughout the mouse brain. As summarized in Table 1, signal intensities varied from undetected $(-)$, faint $(+)$, moderate $(++)$ and strong $(+++)$. A detailed description of the most relevant findings organized into brain areas is as follows:

\section{Telencephalon}

Different $L R R K 2$ mRNA expression patterns were observed in different cortical areas. In some areas, $L R R K 2$ was expressed throughout the six cortical areas and the signal intensity depended on the cell density in each layer. This was the case of cingularfrontal (Fig 2A), temporal (Fig. 2D) or occipital and the medial prefrontal cortex. In other areas, $L R R K 2$ was absent or in disperse neurons in specific cortical layers. In the hindlimb area of the cortex (Fig. 2B) and in the parietal cortex (Fig. 2C), labelling was specifically scattered into the layers II/III and in layer V.

Within the septal area, LRRK2 mRNA was not expressed in the nucleus triangularis septalis and was weakly expressed in the dorsal, ventral and intermediate parts of the lateral septal nucleus. However, a strong signal was observed in the horizontal limb of the medial septal nucleus, and a less but consistent labelling appeared in its vertical division dorsal tenia tecta. Furthermore, this gene was also highly expressed in the adjacent septohippocampal nuclei (Fig. 3A).

Within the hippocampus, LRRK2 mRNA was abundantly expressed in the pyramidal cell layer of Ammon's horn (CA1, CA2 and CA3) as well as in the granular cell layer of the dentate gyrus (Fig. 3B). A strong expression was also observed in disperse neurons either located in the molecular or in the radiatum-oriens layers. Strong labelling was also observed in the hilar region of the dentate gyrus.

$L R R K 2$ mRNA was strongly expressed in almost every part of the amygdala except for the cortical-superficial area (Fig 3C). Thus, it was strongly expressed in the amygdaloid 
intercalary gray area, the amygdalostriatal transition area, and it was moderately expressed in the amygdalopiriform transition area. With regard to the basal amygdaloid nucleus, a very strong LRRK2 expression was observed in the lateral, basomedial, basolateral and posterior basal divisions (Fig. 3D). A strong LRRK2 expression was noted in the medial amygdaloid nucleus in both the posterodorsal and posteroventral parts. $L R R K 2$ expression was also detected in the intraamygdaloid division of the bed nucleus of the stria terminalis close to the posterodorsal area of the medial amygdaloid nucleus and also in the central amygdala. The cortical nucleus, located proximally to the medial amugdaloid nucleus, also expresses LRRK2 mRNA in both the posterolateral and posteromedial parts. No signal was seen in the nucleus of the lateral olfactory tract or in the cortical amygdala (Fig. 3C)

$L R R K 2$ mRNA expression was high in the most superficial layers of the piriform cortex (Fig. 3E). The olfactory tubercule and the islands of Calleja are located close to the piriform cortex, which also showed strong LRRK2 expression (Fig. 3F).

Within the basal ganglia area, LRRK2 mRNA was detected throughout the striatum, whereno patchy differences were observed that can indicate a possible differential reaction between striosome and matrix. Interestingly, the globus pallidus was nearly devoid of any LRRK2 expression (Fig. 4A).

\section{Diencephalon}

With the exceptions of paraventricular thalamic nucleus (Fig. 4B) and the reticular nucleus (Fig 4C), which strongly expresses LRRK2 mRNA, all the nuclei within the thalamus moderately expressed this gene (Fig. 4C). Some labelled cells were observed in both the medial and lateral geniculate nuclei.

A strong LRRK2 mRNA expression was also observed in the medial habenular nucleus. However, only a very faint expression could be seen in the lateral nucleus (Fig. 4B). The zona incerta also contained strongly labelled neurons (Fig 3C).

With regard to the hypothalamus, an $L R R K 2$ mRNA expression was observed in the posterior and dorsal areas, although its expression was very weak in the ventrolateral nucleus. The ventromedial hypothalamic nucleus showed a moderate-to-high expression in the dorsomedial and central parts but not in the ventrolateral one (Fig. 4D). The dorsomedial hypothalamic nucleus showed a high LRRK2 mRNA expression in compact, ventral and dorsal parts (Fig. 4D). A scattered reaction was observed in the lateral hypothalamic area. 
In addition to these above-mentioned diencephalic areas, the geniculate, zona incerta and subthalamic nucleus also expressed LRRK2 moderately.

\section{Brainstem}

A strong $L R R K 2$ mRNA expression was observed in both the dorsal and ventral tiers of substantia nigra pars compacta.In contrast, weak LRRK2 mRNA expression was detected throughout the substantia nigra pars reticulata (Fig. 4E).

The ventral tegmental area and the nucleus of Darkschewitsch moderately expressed LRRK2 mRNA. A strong reaction was observed in the intermediate gray layer of the superior colliculus (Fig 4E) and the red nucleus. Moreover, dispersely labelled neurons also occurred in the inferior colliculus and the nuclei of the lateral lemniscus.

The periaqueductal gray also showed disperse staining (Fig. 4E). Moderate labelling was detected in the dorsal and in the median raphe nucleus. In contrast, the dorsal tegmental nuclei were almost devoid of expression.

Faint $L R R K 2$ mRNA expression was seen in the anterior, posterior and medial pretectal nuclei. Disperse labelling was observed along the reticular formation whereas some cells of the pontine nuclei also expressed $L R R K 2$.

\section{Cerebellum}

As seen in figure $4 \mathrm{~F}$ the granular cell layer of the cerebellum exhibited a moderate LRRK2 mRNA expression. On the other hand, neither the Purkinje cell layer nor the basal nuclei showed such mRNA expression. 


\section{DISCUSSION}

Previously, Northern blot analysis showed that LRRK2 is expressed in the cerebellum, cerebral cortex, medulla, spinal cord, occipital pole, frontal lobe, temporal lobe and putamen (Paisan-Ruiz, et al., 2004). However, a complete and detailed map of LRRK2 mRNA localization in the central nervous system was still lacking. Thus, with the objective of providing a complete atlas of the LRRK2 mRNA distribution, we used in situ hybridization analysis with digoxigenin-labelled RNA probes on mice brain coronal sections. The results clearly show that $L R R K 2 \mathrm{mRNA}$ is distributed throughout the mouse brain. However, a stronger hybridization signal appears in specific brain regions such as the deep cerebral cortex layers, cingulate cortex, piriform cortex, Ammon's horn and dentate gyrus, caudate putamen, substantia nigra, some parts of the amygdala, reticular thalamic nucleus or cerebellar granular cell layer. Amongst these high LRRK2 expression areas, the caudate putamen, the substantia nigra pars compacta and the granular cell layer of the cerebellum, are involved in the regulation of motor activity. As most clinical PD symptoms are related to motor dysfunction, this expression data may indicate a role of $L R R K 2$ in motor activity regulation. Regarding the appearance of LRRK2 mRNA in the granular cell layer of the cerebellum, currently cerebellar symptoms have not been described in PD patients. Interestingly, several studies have related the appearance of parkinsonian motor features (parkinsonism) to the expansion of CAG repeats encoding polyglutamine tracts within Spinocerebellar Ataxia-related genes (Gwin-Hardy et al., 2000; Simon-Sanchez et al., 2005; Wu et al., 2004). The phenotype of these Spinocerebellar Ataxia patients is variable and may cause a disease that clinically overlaps with Parkinson's disease.

Other structures indirectly related to motor activity regulation also express LRRK2 mRNA. We detected strong hybridization signals in the hippocampus, piriform cortex, cingulate cortex, olfactory tubercle, amygdala or reticular thalamic nucleus. Potential relationships between these expression areas and PD pathology have been suggested by several groups studying PD and atrophy. Patients with demented PD showed gray matter volume decreases in several brain regions such as the putamen, accumbens nuclei, some parts of the thalamus, hippocampus or cingulate cortex. Moreover, Camicioli et al (2003) found that a hippocampus atrophy was more often displayed in PD patients than in healthy subjects regardless of any existing cognitive dysfunction. In relation to the amygdala, Junque et al (2005) found that both demented and nondemented PD patients have clear amygdalar and hippocampal atrophies. Since the 
amygdala and the hippocampus are key structures of the limbic system, LRRK2 may play a role in this function. In this respect, it is interesting to note the absence of cognitive dysfunction in most PD patients with dardarin mutations (for example see Paisan-Ruiz et al, 2005).

As we have previously mentioned, we also found a strong LRRK2 mRNA expression in the olfactory tubercle and some other olfactory related areas, such as the piriform cortex. It could be speculated from this finding that dardarin might play a role in the known loss of smell in idiopathic PD (Berendse et al, 2001).

One important finding from this work that may be relevant to the etiopathogenesis of $\mathrm{PD}$, is the strong LRRK2 expression in the putamen which contrasts with a lower or non-signal in the globus pallidus. We can speculate that compensatory mechanisms between both areas might be disrupted as a consequence of dardarin dysfunction.

Wszolek and colleagues reported the finding of different pathological signatures in brain tissue from four members of a PD family with the R1441C dardarin mutation. These authors found Lewy body pathology in two of the four individuals. One of the two Lewy body positive patients also had tau inclusions. A third patient of this family had solely tau inclusions whereas a fourth one only showed neuronal loss and gliosis in the substantia nigra as found in the other three cases (Wszolek, et al., 2004). Our results are consistent with this observations in that LRRK2 is expressed in all the areas where Wszolek and colleagues found evidence of pathological lesions. Moreover, Gai et al. (2005) have recently reported the presence of dardarin in Lewy bodies, neurofibrillary tangles and dystrophic neurites in PD, diffuse Lewy body disease, Alzheimer's disease and Multiple System Atrophy suggesting that dardarin could be involved in mechanisms leading to neurodegeneration, at least within these disorders.

In summary, this study demonstrates the existence of LRRK2 mRNA in many brain regions and nuclei. These results suggest that mutations in LRRK2 could affect several motor structures that playing an important role in PD development. In accord with this, most brain regions involved in the nigrostriatal pathway abundantly express $L R R K 2$ mRNA. The discovery of a strong LRRK2 expression in structures such as the hippocampus, amygdala or olfactory tubercle, may indicate that LRRK2 dysfunction leads to the appearance of the non-motor symptoms that are characteristic of PD. 


\section{ACKNOWLEDGMENTS}

This work was supported by grants from the Ministerio de Educación y Ciencia (GEN2001-4851-C06-01) and Fundación Ramón Areces (DI-TER Parkinson) to JP-T. JS-S is a recipient of a CSIC fellowship (I3P) and VH-P is funded by a fellowship from the Ministerio de Sanidad y Consumo (BEFI). JP-T is part of a Generalitat Valenciana Network of Excellence (GRUPOS 2003/0015). The authors are greatly indebeted to Ms

Hellen Warburton and to Dr. Jayanth Chandran for their assistance in editing this manuscript. 


\begin{abstract}
Abbreviations used in figures.
\end{abstract}

\begin{tabular}{|c|c|}
\hline APT & anterior pretectal nucleus \\
\hline Arc & nucleus arcuatus \\
\hline ASt & amygdalostriatal transition area \\
\hline BLA & basolateral amygdala \\
\hline BMA & basomedial amygdala \\
\hline CA1 & field 1 of the Ammon's horn \\
\hline CA3 & field 3 of the Ammon's horn \\
\hline $\mathrm{CeA}$ & central amygdala \\
\hline $\mathrm{Cg}$ & cingular cortex \\
\hline ci & capsula interna \\
\hline $\mathrm{CoA}$ & cortical amygdala \\
\hline DG & dentate gyrus \\
\hline DHA & dorsal hypothalamic area \\
\hline $\mathrm{dmVMH}$ & dorsomedial division of the ventromedial hypothalamic nucleus \\
\hline EW & edinger westfal nucleus \\
\hline $\mathrm{f}$ & fornix \\
\hline Fr & frontal cortex \\
\hline GP & globus pallidum \\
\hline HL & hindlimb cortex \\
\hline $\mathrm{ICj}$ & islands of Calleja \\
\hline IL & infralimbic medial prefrontal cortex \\
\hline InG & intermediate gray layer of the superior colliculus \\
\hline IP & interpeduncular nucleus \\
\hline LA & lateral amygdala \\
\hline LHA & lateral hypothalamic area \\
\hline $\mathrm{LHb}$ & lateral habenula \\
\hline LOT & nucleus of the lateral olfactory tract \\
\hline $\mathrm{MeA}$ & medial amygdala \\
\hline MGB & medial geniculate body \\
\hline $\mathrm{MHb}$ & medial habenula \\
\hline ot & optic tract \\
\hline PAG & periaqueductal gray \\
\hline Par & parietal cortex \\
\hline PFA & perifornical area \\
\hline Pir & piriform cortex \\
\hline $\mathrm{Pu}$ & putamen \\
\hline PVP & paraventricular posterior thalamic nucleus \\
\hline $\mathrm{RN}$ & red nucleus \\
\hline RSG & retrosplenial granular cortex \\
\hline RT & reticular thalamic nucleus \\
\hline S & subiculum \\
\hline $\mathrm{SNC}$ & substantia nigra pars compacta \\
\hline SNR & substantia nigra pars reticulata \\
\hline $\mathrm{Te}$ & temporal cortex \\
\hline TT & tenia tecta \\
\hline TuMC & tuberal magnocellular division of the lateral hypothalamic area \\
\hline VEnd & ventral endopiriform division of the claustrum \\
\hline vlVMH & ventrlateral division of the ventromedial hypothalamic nucleus \\
\hline VP & ventral pallidum \\
\hline
\end{tabular}


VPL ventral posterolateral thalamic nucleus

ZI zona incerta 


\section{ABBREVIATIONS}

LRRK2: Leucine-rich repeat kinase 2

PD: Parkinson's disease.

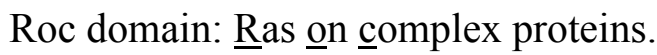

COR domain: $\underline{\mathrm{C}}$-terminal $\underline{\text { of }} \underline{\mathrm{R} o c}$. 


\section{REFERENCES}

Berendse H.W. Booij J. Francot C.M. Bergmans P.L. Hijman R. Stoof J.C. Wolters E.C. (2001) Subclinical dopaminergic dysfunction in asymptomatic Parkinson's disease patients' relatives with a decreased sense of smell. Neurology 50, 34-41

Bosgraaf L, Van Haastert PJ. Roc, a Ras/GTPase domain in complex proteins. Biochim Biophys Acta. 2003 Dec 7; 1643 (1-3): 5-10.

Camicioli R, . Moore M.M., Kinney A,. Corbridge E,. Glassberg K,. Kaye J.A. (2003) Parkinson's disease is associated with hippocampal atrophy. Mov. Disord. 2003 Jul; 18 (7): 784-790.

Funayama M, Hasegawa K, Kowa H, Saito M, Tsuji S, Obata F. A new locus for Parkinson's disease (PARK8) maps to chromosome 12p11.2-q13.1. Ann Neurol. 2002 Mar; 51 (3): 296-301.

Gai W. Wu C. Chilcote T.J. Anderson J.P. Schlossmacher M.G. Blumbergs P.C. Jensen P. Halliday G.M. (2005) LRRK2 in neural inclusions in Parkinson's, Alzheimer's, and related diseases. Program No. 1007.6. $35^{\text {th }}$ Annual Meeting of the Society for Neuroscience. Washington, DC

Gwinn-Hardy K, Chen JY, Liu HC, Liu TY, Boss M, Seltzer W, Adam A, Singleton A, Koroshetz W, Waters C, Hardy J, Farrer M. Spinocerebellar ataxia type 2 with parkinsonism in ethnic Chinese. Neurology. 2000 Sep 26; 55(6): 800-5.

Junque C., Ramirez-Ruiz B., Tolosa E., Summerfield C., Marti M.J., Pastor P,. GomezAnson B., Mercader J.M.. (2005) Amygdalar and hippocampal MRI volumetric reductions in Parkinson's disease with dementia. Mov. Disord. 2005 May; 20 (5):, 540-544.

Paisan-Ruiz C, Jain S, Evans EW, Gilks WP, Simon J, van der Brug M, Lopez de Munain A, Aparicio S, Gil AM, Khan N, Johnson J, Martinez JR, Nicholl D, Carrera IM, Pena AS, de Silva R, Lees A, Marti-Masso JF, Perez-Tur J, Wood NW, Singleton AB. Cloning of the gene containing mutations that cause PARK8linked Parkinson's disease. Neuron. 2004 Nov 18; 44 (4): 595-600.

Paisan-Ruiz C, Saenz A, Lopez de Munain A, Marti I, Martinez Gil A, Marti-Masso JF, Perez-Tur J. Familial Parkinson's disease: clinical and genetic analysis of four Basque families. Ann Neurol. 2005 Mar; 57 (3): 365-72.

Simon-Sanchez J, Hanson M, Singleton A, Hernandez D, McInerney A, Nussbaum R, Werner J, Gallardo M, Weiser R, Gwinn-Hardy K, Singleton AB, Clarimon J. 
Analysis of SCA-2 and SCA-3 repeats in Parkinsonism: evidence of SCA-2 expansion in a family with autosomal dominant Parkinson's disease. Neurosci Lett. 2005 Jul 1-8; 382 (1-2): 191-4.

Summerfield C, Junque C, Tolosa E, Salgado-Pineda P, Gomez-Anson B, Marti MJ, Pastor P, Ramirez-Ruiz B, Mercader J. Structural brain changes in Parkinson disease with dementia: a voxel-based morphometry study. Arch Neurol. 2005 Feb; 62 (2): $281-5$.

Wszolek Z.K. Pfeiffer R.F. Tsuboi Y. Uitti R.J. McComb R.D. Stoessl A.J. Strongosky A.J. Zimprich A. Muller-Myhsok B. Farrer M.J. Gasser T. Calne D.B. Dickson D.W. (2004) Autosomal dominant parkinsonism associated with variable synuclein and tau pathology. Neurology 62, 1619-1622.

Wu YR, Lin HY, Chen CM, Gwinn-Hardy K, Ro LS, Wang YC, Li SH, Hwang JC, Fang K, Hsieh-Li HM, Li ML, Tung LC, Su MT, Lu KT, Lee-Chen GJ. Genetic testing in spinocerebellar ataxia in Taiwan: expansions of trinucleotide repeats in SCA8 and SCA17 are associated with typical Parkinson's disease. Clin Genet. 2004 Mar; 65 (3): 209-14. 
Table 1. Relative levels of expression of $L R R K 2$ in adult mouse brain

Brain area

(I)Telencephalon

Cerebral cortex

Olfactory tubercle

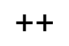

$++$

$+++$

Claustrum

\section{Hippocampal formation}

Ammon's horn

Dentate gyrus

$+++$

$+++$

Septal area

Tenia tecta

vertical Medial Septal Nucleus

horizontal Medial Septal Nucleus

Lateral Septal Nucleus

Septohippocampal Nucleus

Nucleus Triangularis Septalis

Amygdala and extended amygdala

Amygdaloid intramedullary gray

Amygdalopiriform transition area

Amygdalostriatal transition area

Basolateral amygdaloid nucleus

Basomedial amygdaloid nucleus

Central amygdaloid nucleus

Medial amygdaloid nucleus

Lateral amygdaloid nucleus

Nucleus of the lateral olfactory tract

Cortical amygdala

Bed nucleus of the stria terminalis

Basal ganglia

Globus pallidus

Caudate putamen

Nucleus accumbens

\section{(II) Diencephalon}

Thalamus

Anterodorsal nucleus

Reticular nucleus

Parafascicular nucleus

Mediodorsal nucleus

Centrolateral nucleus

Paraventricular nucleus

\section{Habenular complex}

Medial nucleus

Lateral nucleus

Subthalamus
$++$

$++$

$+$

$++$

$+++$

$++$

$++$

$+$

$++$

$+$

$++$

$+++$

$++$

$+++$

$+++$

$+++$

$+$

$+++$

$++$

$-$

$++$

$+++$

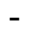

$+++$

$-$

$++$ 
Zona incerta

Hypothalamus

Preoptic nuclei

Posterior area

Dorsal area

Ventrolateral nucleus

$+$

Ventromedial nucleus

$+$

Dorsomedial nucleus

(III) Brainstem

Pretectum

Ventral tegmental area

Red nucleus

Nucleus of Darkschewitsch

VTA

$++$

Periaqueductal Gray

$+$

$++$

Median Raphe

Dorsal Raphe

$++$

$++$

Dorsal tegmental nuclei

Pontine nuclei

$$
-
$$

$++$

Superior colliculus

$++$

Inferior colliculus

\section{Substantia nigra}

Pars compacta

Pars reticulata

\section{(IV) Cerebellum}

Cerebellar nuclei

Cortex

Purkinge cell layer

Granule cell layer

$++$ 
Figure legends

Figure 1.- LRRK2 mRNA expression in the mouse brain. Note that the antisense probe (A) shows a specific hybridization pattern and that the sense probe (B) show no hybridization signal. The hybridization pattern observed after $2 \mathrm{~h}$ of developing time is shown (C).

Figure 2.- LRRK2 mRNA expression in different cortical areas, (A) cingular/frontal cortical areas, (B) hindlimb cortical area, (C) parietal cortex, and (D) temporal cortex. Note that in both the hindlimb and parietal cortices, layers II/III and V showed scattered labelling. A long (12h) chromogenic reaction was performed in all these cases. Calibration bar $200 \mu \mathrm{m}$.

Figure 3.- LRRK2 mRNA distribution in telencephalic areas. (A) Rostral telencephalic areas tenia tecta and infralimbic medial prefrontal cortex. (B) Hippocampus, note that the nonpyramidal neurons of the Ammons horn (arrows) as well as the hilar neurons also express LRRK2. (C) and (D) Amygdala, note the scattered labelling in cortical amygdala and the nucleus of the lateral olfactory tract the square in $\mathrm{C}$ as magnified in D. (E) Piriform cortex, note the strong reaction of the superficial layer. (F) Rostral ventral telencephalon showing the islands of Calleja and the ventral striatum. A long (12h) chromogenic reaction was performed in all these cases. Calibration bar $200 \mu \mathrm{m}$.

Figure 4.- LRRK2 mRNA distribution in subcortical areas. A) Striatum, note the strong labelling in the putamen and contrastingly, the specific scattered reaction in the globus pallidus. B) habenula and paraventricular thalamic nucleus, contrasting the high signal in the medial habenula, the lateral habenula only showed disperse cells. C) Reticular thalamic nucleus in the case of a short (2h) chromogenic reaction. D) Ventral tuberal region of the hypothalamus, note the scattered labelling in the ventrolateral division of the ventromedial hypothalamic nucleus contrasting with the rest of the hypothalamic region and areas. E) Midbrain, note the strong reaction in the substantia nigra pars compacta and in some midbrain nuclei including the interpeduncular and red nuclei as well as the periaqueductal gray and superior colliculus. F) Cerebellum, note the strong reaction in the granular layer. A long (12h) chromogenic reaction was performed in all the cases except C. Calibration bar $200 \mu \mathrm{m}$. 

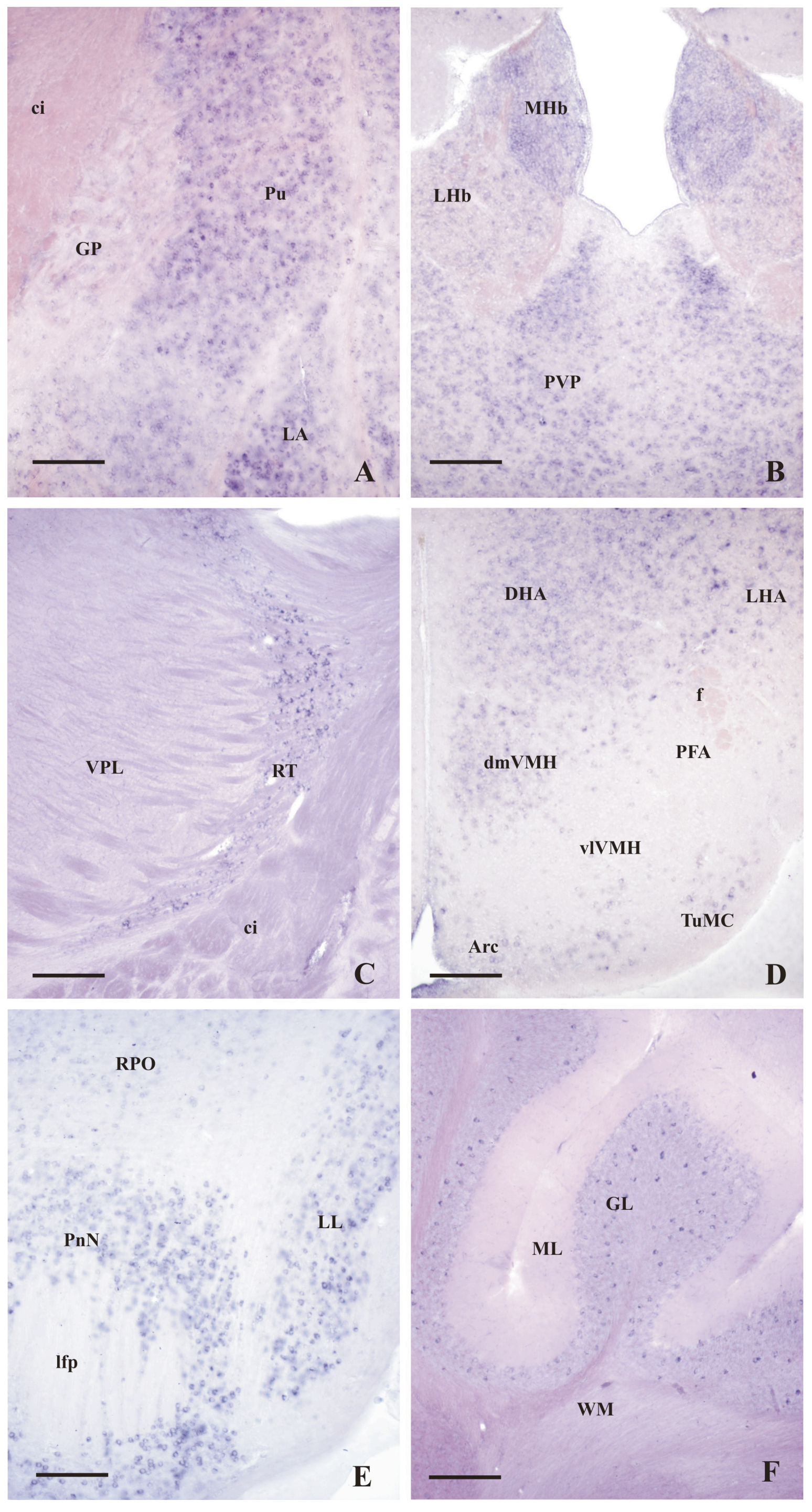


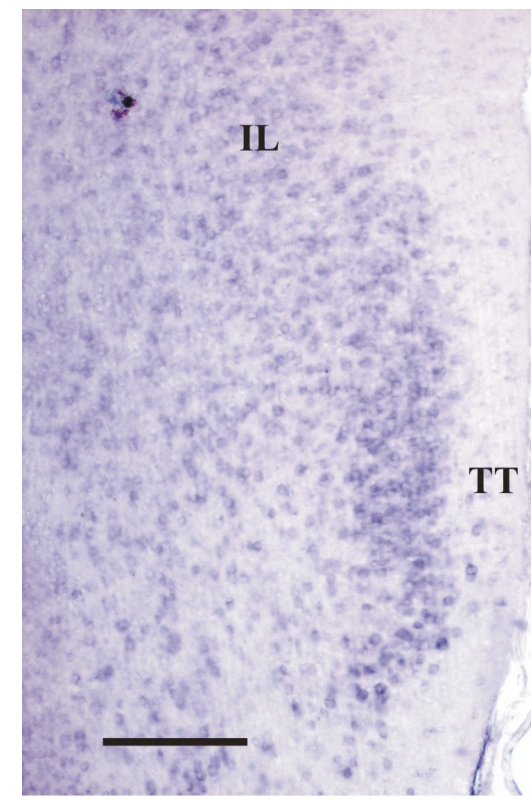

ZI

ci

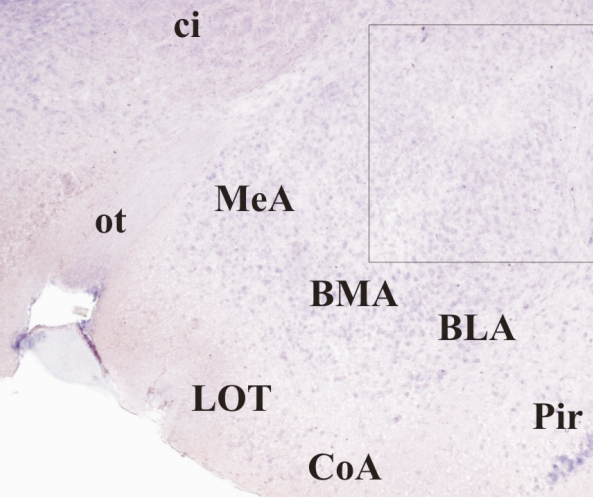

Pu

Pir
Te

A

T

C
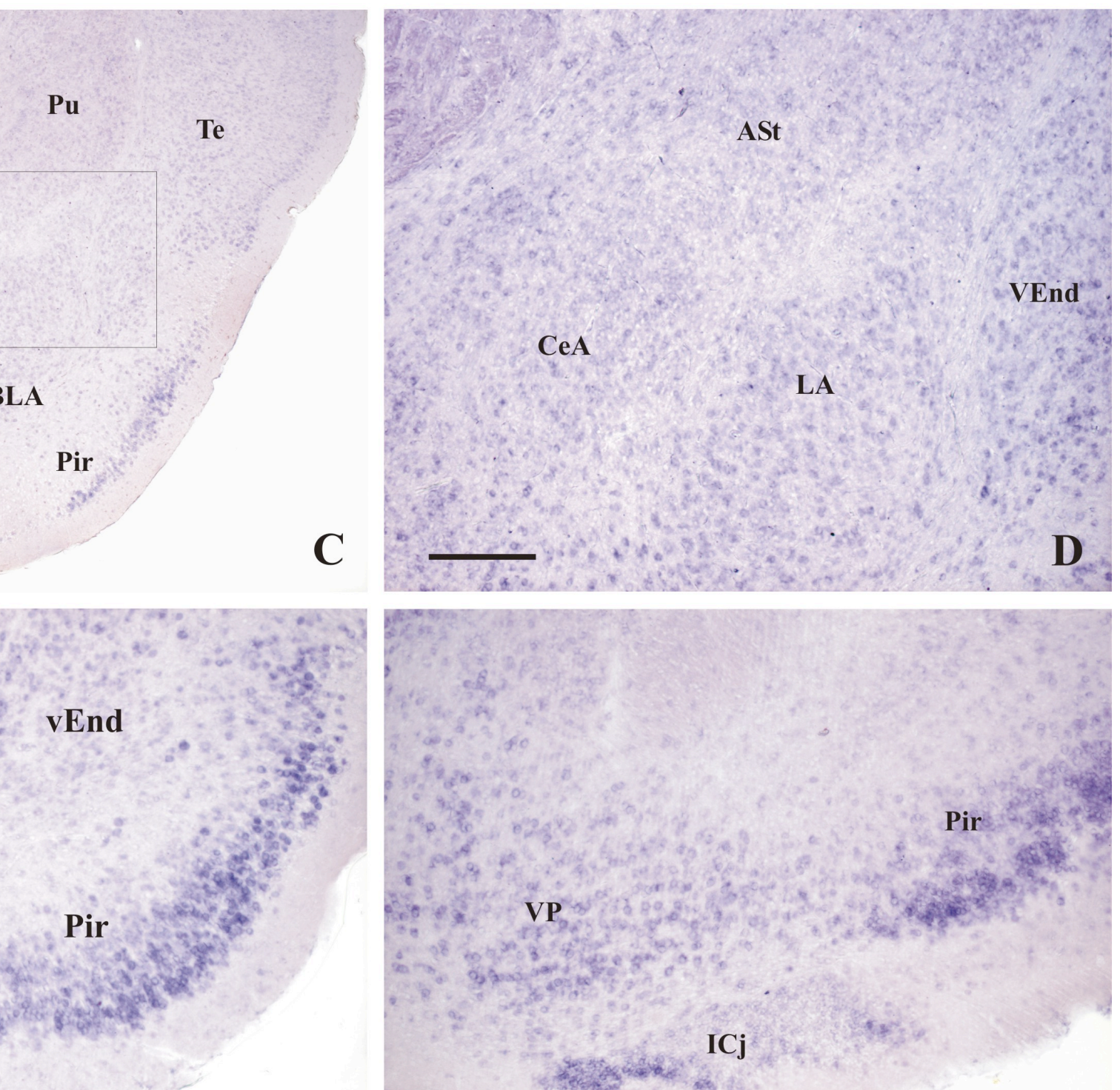

vEnd

Pir

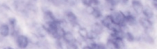

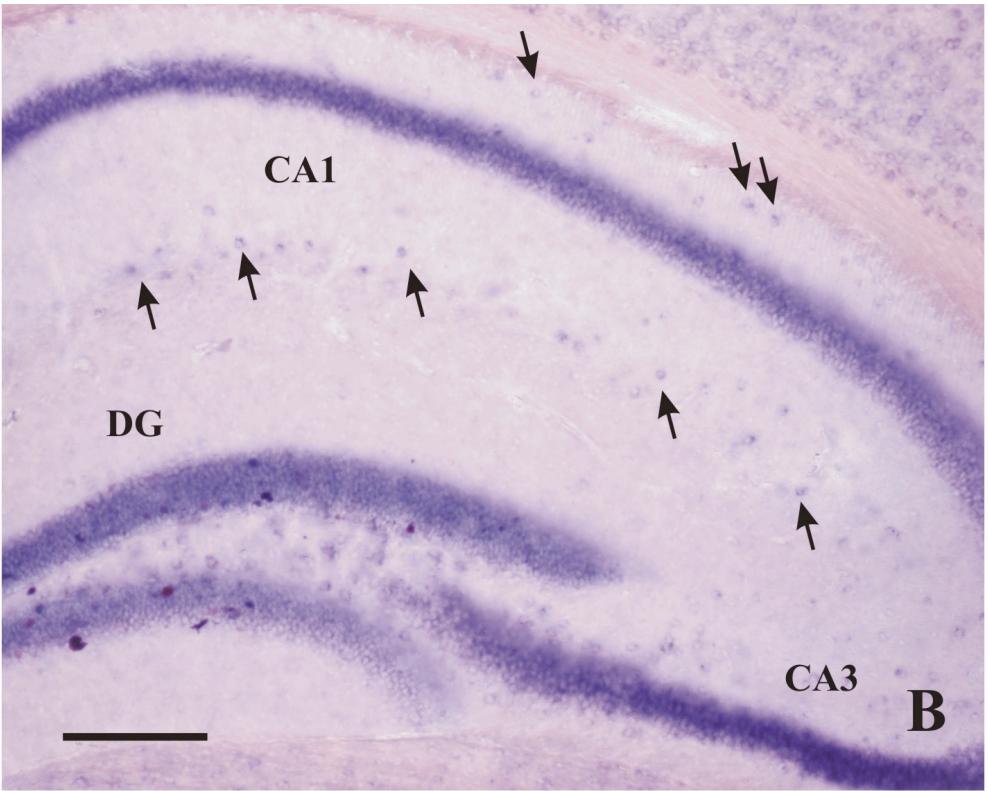

24. $x^{2}$.

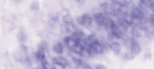

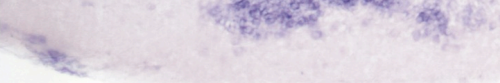



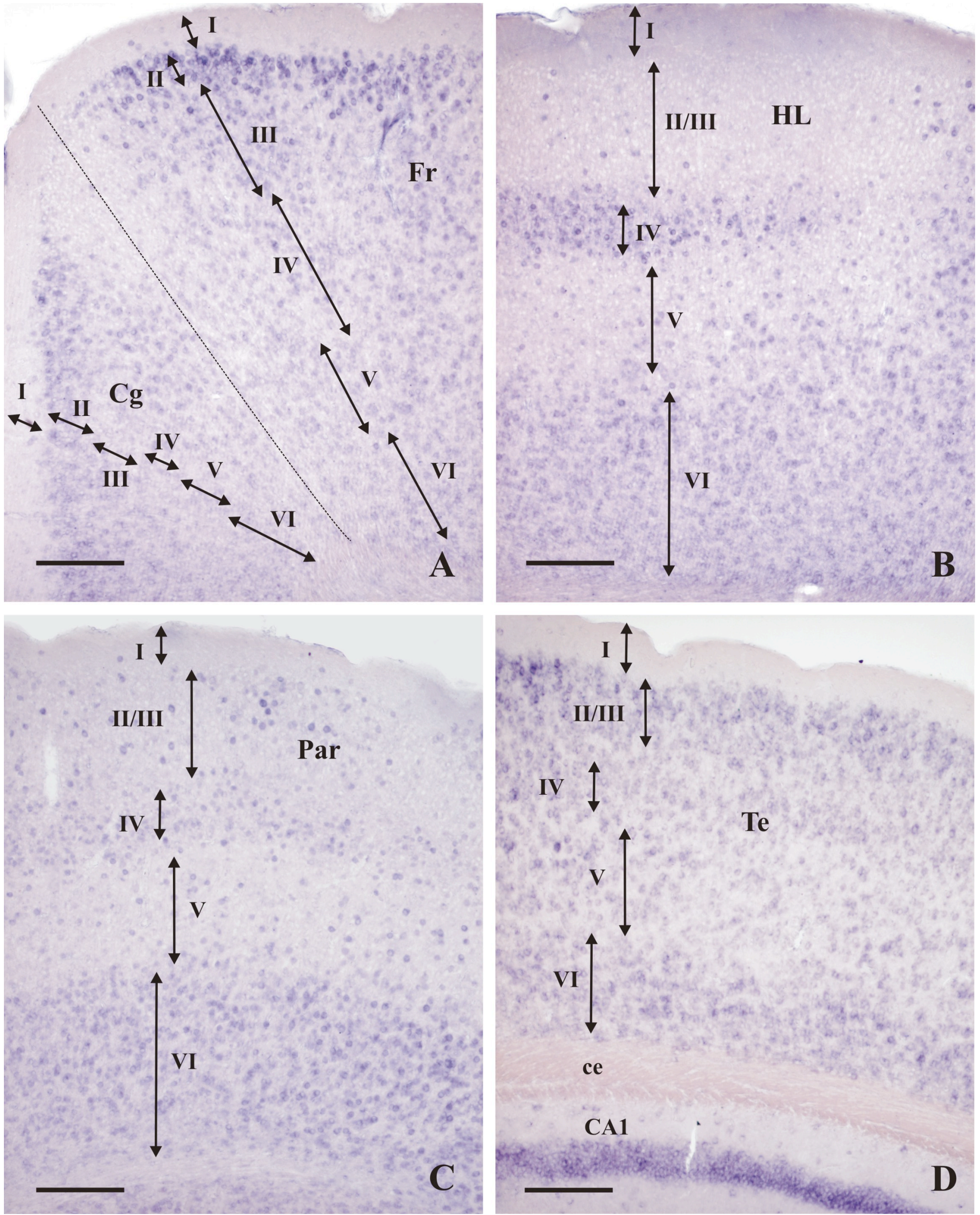

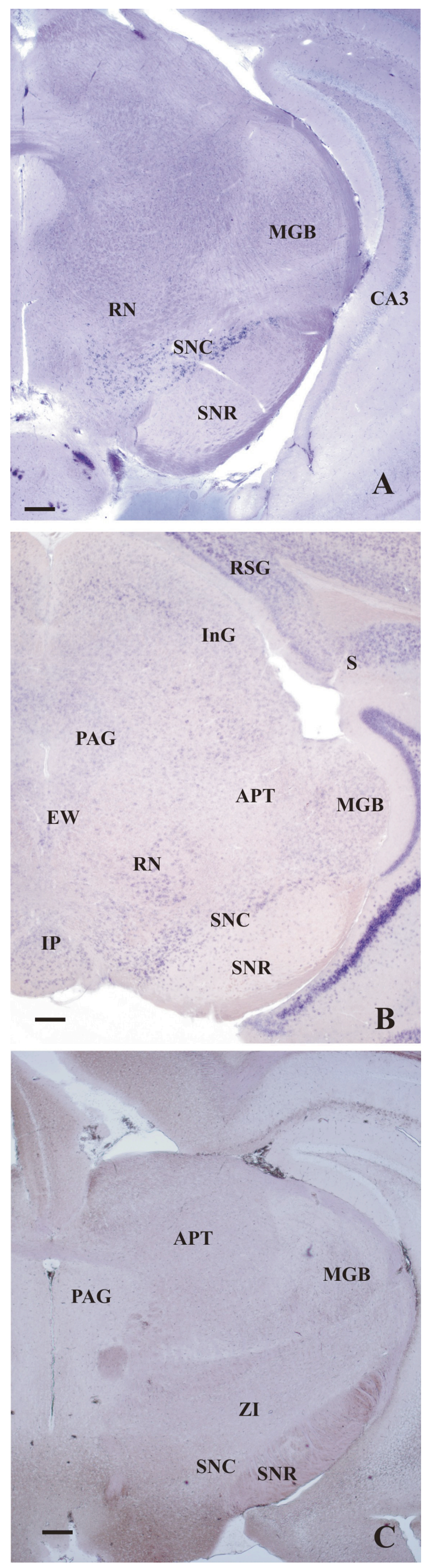